\title{
Nuevo sistema de geolocalización en Navarra para disminuir los tiempos de respuesta en aviso urgente en zonas de montaña y de gran dispersión
}

\section{New geolocation system in Navarre to reduce urgent response times in mountainous and dispersed areas}

\author{
J.M. Ablitas Muro ${ }^{1,2}$, P. González Lorente ${ }^{3,4}$, A. Goienetxe ${ }^{1}$, A. Istúriz ${ }^{3,4}$, J. Biurrun ${ }^{1,2}$, \\ L. Casadamón ${ }^{4}$, M. Pascual ${ }^{1}$
}

\section{RESUMEN}

La identificación de todos los avisos urgentes a domicilio en zonas de montaña y dispersas a través del sistema 112 habitualmente se realiza por el nombre propio del caserío o vivienda, lo que hace imposible localizarlos con los medios GPS habituales. El objetivo que se persiguen en este trabajo es tener un sistema de geolocalización válido en urgencias extra-hospitalarias adaptado a la nomenclatura y geografía de la zona, que permita su implantación en todos los dispositivos móviles sanitarios, consiguiendo una precisión exacta sin pérdidas en el desplazamiento con el fin de evitar un aumento de la morbi-mortalidad por la demora en la atención realizada por profesionales conocedores o no de la zona y que sea extrapolable a otros puntos de la geografía de Navarra o nacional.

Se ha conseguido crear una herramienta de geolocalización adaptada a la orografía y nomenclatura específica de la zona piloto elegida (el valle del Baztán) y se ha hecho compatible con los software de navegación actuales en las dos modalidades posibles, con y sin cobertura de datos móviles (on line y off line). Este sistema se ha integrado en los móviles sanitarios no corporativos mediante archivos KML y se ha aplicado a los 913 caseríos geolocalizados, verificando su utilidad real en los avisos domiciliarios urgentes registrados entre los meses de mayo y septiembre del 2012. Se ha conseguido llegar al caserío (encaminamiento o enrutamiento) demandante de la atención urgente sin pérdidas en carretera o camino forestal independientemente de las características de acceso a éste, porque previamente se tiene registrada la localización del destino en estos archivos (ésta es la gran diferencia con los sistemas GPS actuales).

Palabras clave. Urgencia. Geolocalización. Encaminamiento. GPS. Isócrona.

\begin{abstract}
Identification of all urgent home alerts in mountainous and dispersed areas through the 112 system is normally done using the name of the farmhouse or dwelling, which makes it impossible to localize them with normal GPS means. The aim of this article is to obtain a valid geolocation system in out-of-hospital emergencies that is adapted to the nomenclature and geography of the area, and that can be implanted in all mobile health devices. This will provide exact precision without any loss of time in journeys, so as to avoid an increase in morbidity and mortality due to delays in attention given by professionals, whether or not they know the area. It should be possible to extrapolate this system to other points in the geography of Navarre and Spain.
\end{abstract}

A geolocation tool was created adapted to the orography and specific nomenclature of the chosen pilot area (the Baztan valley) and it was made compatible with the two possible modes of current navigational software, with and without mobile data coverage (on line and off line). This system was integrated into noncorporative health mobile phones using KML archives and applied to the 913 geolocated farmhouses, with its real utility verified in the urgent home alerts registered between the months of May and September 2012. It was possible to reach the farmhouse (routing) requiring urgent attention without getting lost on the road or forest route, irrespective of access characteristics, because the localization of the destination had been registered beforehand in these files (this is the great difference with current GPS systems).

Key words. Emergency. Geolocation. Routing. GPS. Isochrone.
1. Servicio de Urgencia Rural. Servicio Navarro de Salud-Osasunbidea.

2. Instructor de Soporte Vital Avanzado.

3. Especialista en Medicina Familiar y Comunitaria.

4. Servicio Normal de Urgencias. Servicio Navarro de Salud-Osasunbidea.
Recepción: 16 de octubre de 2012

Aceptación provisional: 6 de noviembre de 2012 Aceptación definitiva: 17 de diciembre de 2012 


\section{INTRODUCCIÓN}

El valle del Baztán es una zona situada en el norte de Navarra cuya extensión es de $373,55 \mathrm{~km}^{2}$. Es el municipio más extenso de Navarra y tiene 8.075 habitantes la gran mayoría dispersos en más 15 localidades y numerosos barrios y caseríos o viviendas diseminadas, cuya lengua vernácula es el euskera. Los profesionales sanitarios que desarrollan su trabajo en la zona rural del valle del Baztán, se percatan de la gran dificultad que supone localizar muchos de los caseríos dispersos y viviendas situadas en núcleos poblacionales de nuestro entorno de trabajo. La especial orografía unida a esa dispersión, característica igualmente en otras zonas de territorio rural, supone una dificultad añadida muy importante a la hora de prestar una atención de calidad. La actual inexistencia de mapas precisos y la inefectividad de los GPS, por la no concordancia entre el sistema de localización de éstos y la nomenclatura autóctona usada por nombres propios de caseríos aplicada en las distintas poblaciones, dificulta un óptimo acceso del sanitario al paciente demandante y ofrecer una correcta cobertura sanitaria ajustada a las isócronas sanitarias recomendadas, entendiendo por isócrona, "el tiempo que transcurre en recibir atención un paciente/usuario en un aviso urgente o emergente, por los profesionales sanitarios".

Esta adversidad resulta más manifiesta en la atención a pacientes de carácter grave. Si a esto le añadimos la ansiedad que produce el desconocimiento de la localización del lugar del aviso, la pérdida de cobertura de los teléfonos móviles y la necesidad de tener que utilizar la telefonía fija, provoca inevitablemente la demora en la llegada a una atención urgente domiciliaria.

Este modelo consiste en generar y aplicar, utilizando las nuevas herramientas informáticas, un sistema útil y accesible de localización de puntos, que permita a los profesionales sanitarios atender en el mínimo tiempo posible a los usuarios/pacientes de los distintos servicios de salud, evitando las limitaciones de la navegación GPS tradicional. En el modelo piloto trabajado se han geolocalizado caseríos y viviendas en el Valle del Baztán, permitiendo el sistema ser extendido a otras zonas de Navarra o limítrofes.

El principal problema de la localización GPS es que los sistemas GPS localizan puntos a través de la inserción de calles y números de portal. Estos sistemas son empleados por la mayoría de los sistemas GPS de mayor utilización: TomTom ${ }^{\circledR}$, Sygic $^{\circledR}$, google navigator ${ }^{\circledR}$ etc. Tampoco se libran de estas limitaciones, sistemas de geolocalización como SITNA (sistema cartográfico del gobierno de Navarra) o navegadores tipo GARMIN ${ }^{\circledR}$, que si bien son capaces de localizar puntos a través de calles y números, incluso de coordenadas cartográficas, e introducir ortofotos en sus productos, no resuelven el principal problema de localización que en muchos casos se da en núcleos poblacionales rurales y caseríos apartados. Estos sistemas no sólo no son válidos, sino que en muchas ocasiones pueden inducir a error y retrasar la atención urgente a menos que se tenga un amplio conocimiento geográfico de la zona. Habitualmente los caseríos, casas o lugares son conocidas en la zona por nombres de los propietarios o de parajes especiales y no por el nombre de las calles. En determinados casos se puede solicitar la guía hasta la vivienda por familiares o vecinos, con grandes limitaciones en los casos en los que la persona a atender viva sola o en caso de población envejecida. En otras ocasiones se pueden preguntar las localizaciones a los viandantes, con la consiguiente limitación en los casos de atenciones urgentes nocturnas. Igualmente se ha podido comprobar que las indicaciones telefónicas pueden provocar retraso en el profesional de no tener referencias muy precisas, que incluso pueden inducirle al equívoco.

Con este nuevo sistema pretendemos evitar muchos de los problemas de accesibilidad a la localización de la urgencia, a los que están sujetos los profesionales que trabajan en la urgencia extra-hospitalaria y en la atención primaria rural. 
El objetivo de este trabajo es la creación un nuevo sistema válido de geolocalización y encaminamiento (en lenguaje de navegación GPS se conoce como enrutamiento) para dotar a todos los profesionales que trabajan en el mundo sanitario extra-hospitalario, conocedores o no de la zona, de una herramienta eficaz para llegar a su destino en las mejores condiciones y en el menor tiempo posible mediante la creación de un sistema de localización de puntos y posterior inserción en todo tipo de navegadores GPS y dispositivos móviles ${ }^{1-3}$.

\section{CÓMO SE CREA UNA SISTEMA VÁLIDO DE GEOLOCALIZACIÓN PARA LA URGENCIA EXTRA- HOSPITALARIA RURAL}

Para la creación de un sistema válido de geolocalización es importante diferenciar dos términos y separarlos de la navegación GPS habitual: geolocalización o georreferenciación y encaminamiento (enrutamiento).

a) La georreferenciación o geolocalización ${ }^{4-6}$ hace referencia al posicionamiento con el que se define la localización de un objeto espacial (representado mediante punto, vector, área, volumen) en un sistema de coordenadas. Este proceso es utilizado frecuentemente en los sistemas de información geográfica. La georreferenciación posee una definición tecno-científica aplicada a la existencia de las cosas en un espacio físico, mediante el establecimiento de relaciones entre las imágenes de ráster o vector sobre una proyección geográfica o sistema de coordenadas. Por ello, la georreferenciación se convierte en central para los modelados de datos realizados por los Sistemas de Información Geográfica (SIG).

b) Encaminamiento (enrutamiento). Establece una ruta entre una dirección origen y una dirección destino. Posibilitaría una evolución de este sistema y se podría utilizar en caso de lugares con diferentes accesos y caminos de llegada o diferentes tipos de vía. No sólo se marca el punto final o de llegada, sino que se define el sitio por el que se tiene que llegar. Se realizan rutas prefijadas en las cuales se pueden planificar puntos o sitios de paso, o rutas especificas (el GPS o el usuario del sistema, siguen las instrucciones fijadas por el interesado o creador de rutas). El encaminamiento es especialmente interesante en aquellos lugares en los que el GPS no resulta operativo o provoca rutas que no son las habitualmente utilizadas por los habitantes de la zona o usuarios de algún punto o establecimiento. Esto permite no solo no tener que saber dónde está el lugar, sino no tener que elegir entre varias rutas posibles eligiendo la más rápida, la más cómoda o la que más se adapte a nuestras necesidades. El proceso de enrutamiento es sencillo, ya que muchos de los sistemas GPS como el utilizado en este trabajo Oruxmaps o Garmin son capaces de recordar y almacenar rutas, siendo estas fácilmente exportables.

\section{Localización de caseríos por coordenadas y creación e integración de los archivos de geolocalización o georreferenciados en los dispositivos móviles ${ }^{7-9}$}

Se ha utilizado imágenes vía satélite de los pueblos y caseríos del valle del Baztán a través de Google maps ${ }^{10}$ (Fig. 1). Con mapas hechos a manos, con nombres de caseríos, por profesionales y personas de la zona se han etiquetado por su nombre habitual a estos caseríos. Cuando hablamos de "nombre habitual", es el nombre concreto del caserío o casa ej: Dorreko Borda (Amaiur), Erronea (Erratzu), Landaldea (Arizkun). Se ha procedido a la geolocalización de coordenadas longitud y latitud ${ }^{11}$ de bordas y casas a través de páginas web que permiten generarlas. 

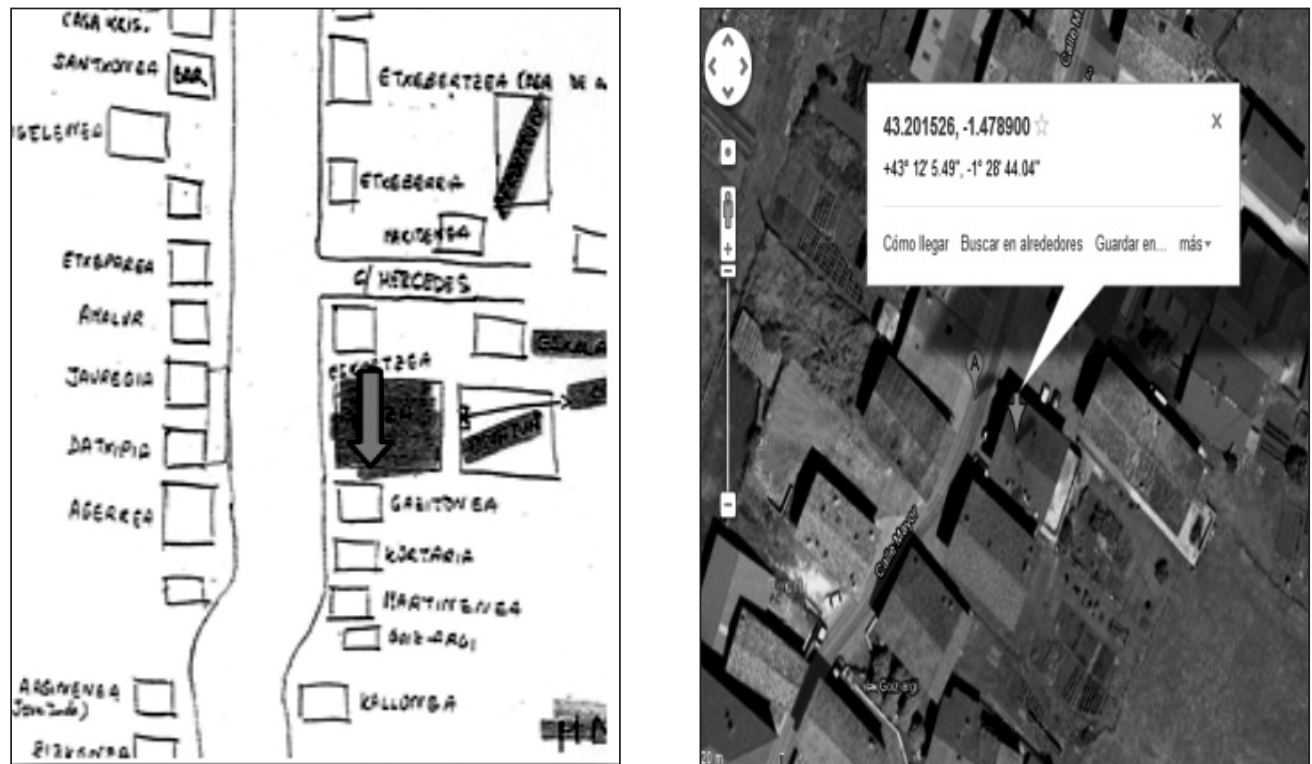

Figura 1. Mapa dibujado y foto de Google maps de Amaiur.

Con esas coordenadas se han generado archivos de datos, cuyo encabezamiento es el nombre del pueblo y el contenido dividido en si el caserío se encuentra en núcleo poblacional o si está diseminado (fuera del pueblo). Igualmente se han especificado los barrios de los diferentes pueblos. Con todo esto se han generado bases de datos en los que existe una correspondencia entre caseríos, pueblo o barrio de pueblo al que pertenecen y sus correspondientes coordenadas. Estas bases de datos se han introducido en Google maps para generar archivos KML para su posterior exportación a distintos dispositivos GPS ${ }^{12}$. Los KML también han sido divididos en pueblos y barrios y se han ordenado todos los caseríos alfabéticamente para que su búsqueda a la hora de utilizar el GPS sea lo más sencilla posible ${ }^{13-15}$. Posteriormente se ha pretendido buscar la forma de utilizar esos archivos de coordenadas, cómo compartirlos y cómo integrarlos en diferentes dispositivos. De esta manera se ha conseguido compartir mapas on line o a través de correos electrónicos, Wathsapp ${ }^{\circledR}$ o SMS de manera que todos los profesionales los tengan. Se han buscado soluciones para integrar los archivos a los diferentes navegadores y dispositivos móviles así como aplicarlo en los diferentes software de navegación. De esta manera se han integrado y probado con resultado satisfactorio en la práctica totalidad de dispositivos y software: Teléfonos móviles con software android ${ }^{\circledR}$, Windows ${ }^{\circledR}$, Iphone ${ }^{\circledR}$ (IOS). Navegadores como TOMTOM $^{\circledR}$ y Garmin ${ }^{\circledR}$. Software de navegación como Windows Navigation ${ }^{\circledR}$, Sigyc $^{\circledR}$, Garmin $^{\circledR}$, Co-pilot $^{\circledR}$ etc.

También se ha utilizado estos archivos en navegadores on line y off line ${ }^{16}$ : sin cobertura de datos móviles o con datos móviles (tarifa de datos $3 \mathrm{~g}$ o Wifi). Por una parte hay navegadores que cargan mapas conforme lo van necesitando, consumiendo datos y otros navegadores ya tienen los mapas incluidos. Esto dos modelos de navegación resultan de vital importancia porque hay dispositivos móviles que no 
tienen tarifa de datos y otros en ocasiones no tienen cobertura $3 \mathrm{~g}$ de telefonía para zonas periféricas alejadas. Este novedoso sistema ha sido utilizado de ambas mane- ras sin depender de la cobertura de datos, sino únicamente del GPS, logrando ver la ruta en todo momento, desde el punto de salida al de llegada (Fig. 2).
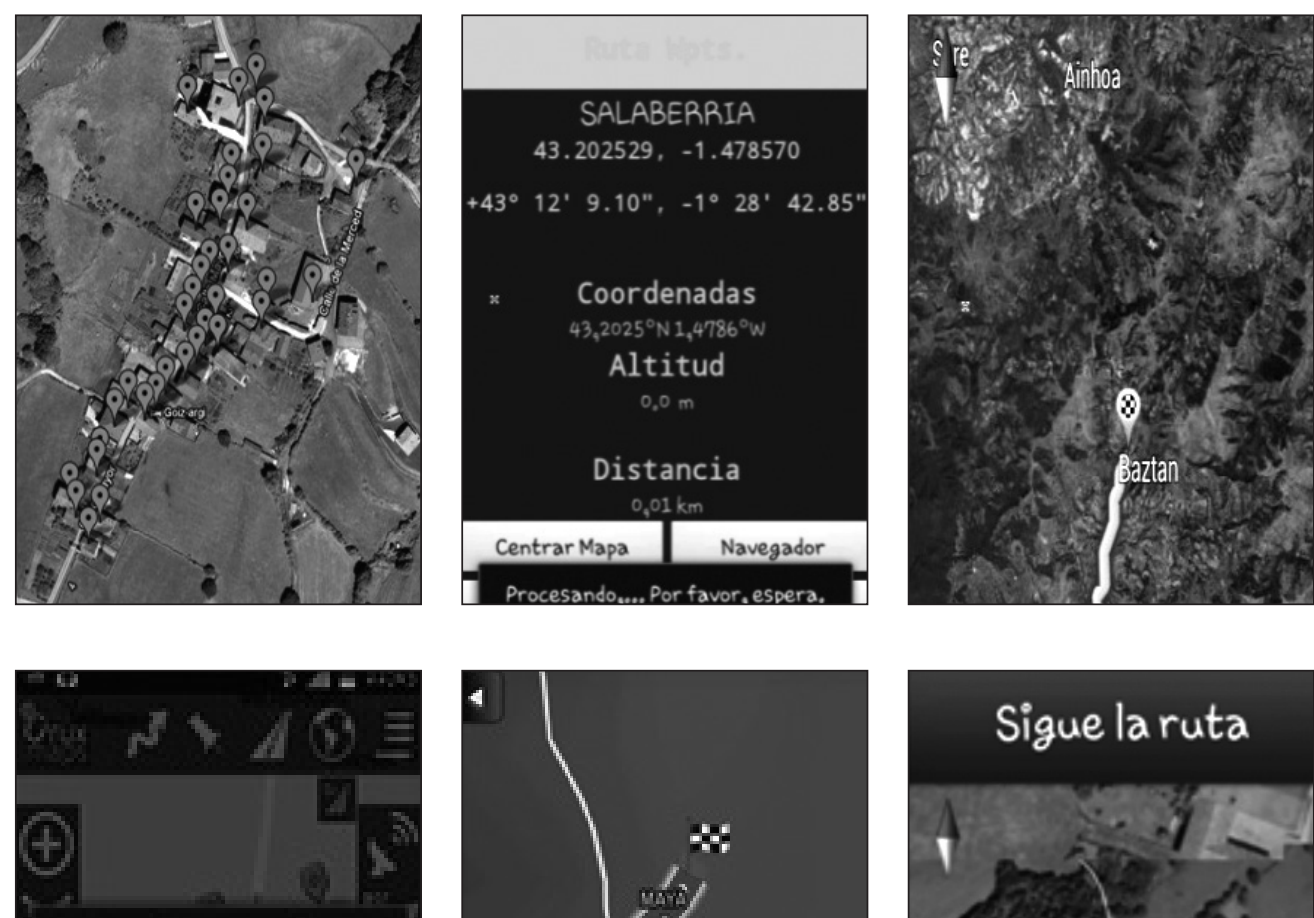

Wo dectinds conplats

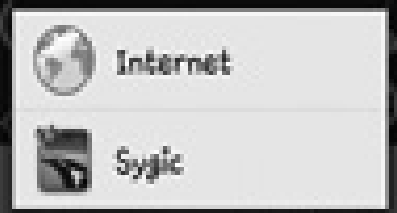

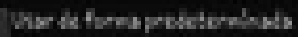
ruthin hes
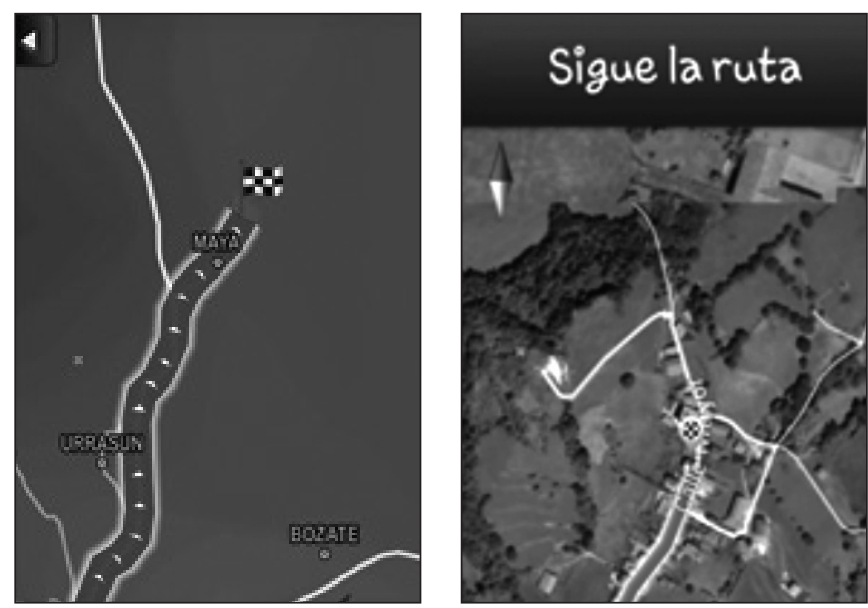

Figura 2. Google Navigator y Sygic: llegando de Amaiur a un caserío desde Elizondo. 


\section{Caseríos geolocalizados y comprobación del sistema creado}

Se han localizado 913 caseríos del valle del Baztán.

Tabla 1. Caseríos en el Valle del Baztán

\begin{tabular}{|c|c|c|c|c|c|c|c|c|}
\hline \multirow[t]{2}{*}{ Amaiur } & Pueblo & 64 & \multirow[t]{5}{*}{ Erratzu } & Pueblo & 97 & \multirow[t]{3}{*}{ Almandoz } & Pueblo & 24 \\
\hline & Diseminados & 40 & & Iñarbil & 11 & & Diseminados & 3 \\
\hline \multirow[t]{6}{*}{ Arizkun } & Pueblo & 76 & & Gorostapolo & 17 & & Mendiola & 1 \\
\hline & Pertalats & 7 & & Iñarbegi & 6 & Aniz & & 47 \\
\hline & Ordoki & 22 & & Untxide & 16 & Arraioz & & 58 \\
\hline & Bergara & 20 & Orabidea & & 25 & Berroeta & & 47 \\
\hline & Bozate & 42 & Ziga & & 32 & Gorramendi & & 4 \\
\hline & Aintzialde & 19 & Zigaurre & & 16 & Lekaroz & & 41 \\
\hline \multirow[t]{2}{*}{ Azpilikueta } & Urrasun & 13 & \multirow[t]{2}{*}{ Irurita } & Pueblo & 86 & \multirow[t]{2}{*}{ Oronoz } & Pueblo & 21 \\
\hline & Apaioa-Zuaztio & 10 & & Regatas & 43 & & Zozaia & 5 \\
\hline \multicolumn{7}{|c|}{ Caseríos Valle del Baztan: pueblos, barrios y diseminados. } & Total & 913 \\
\hline
\end{tabular}

Cada caserío se identifica mediante su coordenada con su nomenclatura real usada en la zona. Se convierten las coordenadas en archivos KML y se integran en los dispositivos móviles ${ }^{16}$ no corporativos de los profesionales que trabajan en la urgencia rural. En situación fuera de urgencia se comprueba la operatividad del sistema creado eligiendo al azar varios caseríos por pueblo geolocalizado, siendo el resultado una precisión exacta en las dos posibilidades on line (con cobertura) off line (sin cobertura) y posteriormente usándose en los avisos externos al centro que los profesionales de urgencias tienen durante los meses de mayo, junio, julio, agosto y septiembre consiguiendo en los avisos registrados llegar al caserío sin extravíos en el desplazamiento (Tabla 2).

Todo lo creado se ha probado con éxito en el sistema operativo android hasta sus últimas versiones icecream 4.0.3 e ios de Apple, en Windows móvil y en los distintos navegadores como Tom Tom ${ }^{\circledR}$. Se han utilizado diferentes software de navegación GPS: IGO ${ }^{\circledast}$, Sygic ${ }^{\circledR}$, Navigon $^{\circledR}$, Co-pilot ${ }^{\circledR}$ pu- diendo ser utilizados en todos ellos. Se han creado archivos KML y comprimidos KMZ para integrar los principales datos de geolocalización. Se han integrado los archivos en los software de navegación, utilizándolos tanto de forma on line como off line. Estos archivos pueden integrarse directamente o utilizando como motor de carga aplicaciones android, como oruxmaps ${ }^{\circledR} \mathrm{o}$ lanzadores de archivos KML como la App kmlmaps ${ }^{\circledR}$ para Apple.

La novedad e interés del sistema es que al ser generado con software libre es totalmente integrable en la práctica totalidad de los dispositivos, no presentando ninguna incompatibilidad que no sea fácilmente subsanable. Otra importante ventaja es que la tecnología necesaria para hacer funcionar el sistema ya está integrada en la gran mayoría de Smartphone, navegadores GPS y tablets, tanto en software (distintos software de navegación, gratuitos o de pago) y hardware (GPS...) abaratando económicamente su aplicación y utilización por dispositivos corporativos de empresas públicas y/o privadas. 
Tabla 2. Nombres y coordenadas de caseríos de cada pueblo geolocalizado

\begin{tabular}{|c|c|c|}
\hline Pueblo & Nombre del caserío & Coordenada \\
\hline Amaiur pueblo & Eskorzea & $43.201782,-1.478699+43^{\circ} 12^{\prime} 6.41^{\prime \prime},-1^{\circ} 28^{\prime} 43.32 ”$ \\
\hline Amaiur dispersión & Alemanea & $43.214382,-1.480279+43^{\circ} 12^{\prime} 51.77^{\prime \prime}, 1^{\circ} 28^{\prime} 49.00^{\prime \prime}$ \\
\hline Arizkun pueblo & Dolatxea & $43.173171,-1.484949+43^{\circ} 10^{\prime} 23.41^{\prime \prime},-1^{\circ} 29^{\prime} 5.82^{\prime \prime}$ \\
\hline Bozate & Atsotenea & $43.173562,-1.484646+43^{\circ} 10^{\prime} 24.82^{\prime \prime},-1^{\circ} 29^{\prime} 4.72^{\prime \prime}$ \\
\hline Urrasun & Agerrea & $43.188583,-1.489146+43^{\circ} 11^{\prime} 18.90^{\prime \prime},-1^{\circ} 29^{\prime} 20.93 ”$ \\
\hline Aintzalde & Bazterretxea & $43.164512,-1.486357+43^{\circ} 9^{\prime} 52.24^{\prime \prime},-^{\circ} 29^{\prime} 10.88^{\prime \prime}$ \\
\hline Errazu & Salaberria & $43.180410,-1.455739+43^{\circ} 10^{\prime} 49.47^{\prime \prime},-1^{\circ} 27^{\prime} 20.66^{\prime \prime}$ \\
\hline Iñarbegi & Aguerrea & $43.135357,-1.440735+43^{\circ} 8^{\prime} 7.29^{\prime \prime},-1^{\circ} 26^{\prime} 26.65 "$ \\
\hline Gorostapolo & Iruin & $43.167169,-1.456686+43^{\circ} 10^{\prime} 1.81^{\prime \prime},-1^{\circ} 27^{\prime} 24.07$ \\
\hline Iñarbil & Ansalas & $43.172709,-1.444485+43^{\circ} 10^{\prime} 21.75^{\prime \prime},-1^{\circ} 26^{\prime} 40.15^{\prime \prime}$ \\
\hline Ordoki & Echebertzea & $43.180200,-1.483063+43^{\circ} 10^{\prime} 48.72^{\prime \prime},-1^{\circ} 28^{\prime} 59.03^{\prime \prime}$ \\
\hline Bergara & Laurentx & $43.169198,-1.492191+43^{\circ} 10^{\prime} 9.11^{\prime \prime},-1^{\circ} 29^{\prime} 31.89^{\prime \prime}$ \\
\hline Irurita pueblo & Olari & $43.13042352,-1.5473864+43^{\circ} 7^{\prime} 49.52^{\prime \prime}, 1^{\circ} 32^{\prime} 50.59 ”$ \\
\hline Ziga & Maiora & $43.119080,-1.570970+43^{\circ} 7^{\prime} 8.69 ”, 1^{\circ} 34^{\prime} 15.49 "$ \\
\hline Pertalax & Xanbarnea & $43.178518,-1.468456+43^{\circ} 10^{\prime} 42.67^{\prime \prime},-1^{\circ} 28^{\prime} 6.44^{\prime \prime}$ \\
\hline
\end{tabular}

\section{Proyecto en constante desarrollo y expansión}

Al ser un proyecto dinámico con cambios constantes debido a la necesidad de estar totalmente actualizado en tiempo y espacio se ha creado un blog: http://rekonditolugar.blogspot.com.es $/{ }^{15}$ en el cual se van ampliando y respondiendo a las dudas que se puedan dar en la utilización de esta tecnología. En el blog se integran las novedades y tutoriales que se van realizando para que el sistema sea un sistema abierto y plural y pueda ser utilizado por todo aquel que lo desee y necesite. Dicho blog también tiene un foro en el que todo el interesado puede exponer sus dudas que serán resueltas, en la medida de lo posible por el creador del sistema. También esta enlazado a través de la red social Facebook para hacer partícipes a los seguidores del blog de la novedades que se puedan dar. http:// www.facebook.com/rekondito.lugar

\section{Hallazgos de esta herramienta}

La localización de puntos GPS se basa en direcciones de calles, códigos postales, puntos de interés (iglesias, centros comerciales, monumentos, museos), resultando dificultoso para determinar puntos con otra nomenclatura o toponimia diferente.

En un aviso urgente se puede tratar de localizar un punto mediante coordenadas triangulando señales de repetidores, siempre que la llamada sea a través del móvil. Estos sistemas, en caso de que los repetidores estén alejados, presentan cierta tasa de error y no ser del todo exactos. Además dependemos de que la llamada se realice desde un teléfono móvil (no de un teléfono fijo) y sin pérdida de cobertura. La localización de lugares a través de telefonía fija se basa en datos telefónicos como: nombre, calle, código postal, número de portal etc. con lo que no soluciona el problema real de localización en zonas dispersas de montaña. 
Con este nuevo sistema de geolocalización para zonas de montaña y de gran dispersión se consigue una disminución de la isócrona de atención en la patología urgente evitando pérdidas de tiempo en la búsqueda del destino, con la consiguiente disminución de la morbi-mortalidad basándonos en criterios de atención al paciente crítico, situación en la cual los minutos son fundamentales para el pronóstico de la supervivencia.

El resultado de este modelo permite la navegación hasta el punto exacto de atención, desde nuestro punto de salida guiados por GPS y evitando la posibilidad de pérdidas y demoras, así como el establecimiento de un mapa exacto de caseríos y localizaciones en el valle del Baztán potencialmente a atender en una urgencia.

Este sistema de gran versatilidad y bajo coste permite ser integrado en la práctica totalidad de los navegadores actuales ya que existen sistemas de conversión de archivos KML para hacer compatibles los datos con los diferentes tipos de navegadores. Nos permite compartir datos de localización con escaso consumo de recursos informáticos y de capacidad de memoria pudiendo ser enviadas las localizaciones a través de correo electrónico, mensaje u otro medio de difusión, incluido sistema de trasferencia de datos móviles con datos tipo whatsapp, icloud, dropbox o google+, etc. Además permite actualizaciones de puntos in situ que no estén previamente localizados a través de coordenadas, como longitud y latitud.

La potencialidad del modelo podría permitir asimismo la posibilidad de adjuntar datos anexos referentes a las personas que viven en un lugar, pudiendo crear vínculos a la historia clínica informatizada e incluso adjuntar el historial clínico de un paciente protegiendo dicha información (con las licencias y permisos oportunos). $\mathrm{Al}$ no necesitar un soporte tecnológico especial, bastaría con un teléfono móvil de empresa con GPS, Tablet o Navegador.

Este sistema permite además múltiples posibilidades de utilización, desde la atención de urgencia sanitaria, hasta su uso por personal no sanitario con desconocimiento de la zona, permitiendo a todos llegar al punto de interés en el menor tiempo posible y optimizar el trabajo de otros colectivos profesionales como son los técnicos de ambulancia, repartidores de oxígeno a domicilio, y otros gremios como pueden ser comerciales o transporte urgente nacional e internacional, protección civil, etc., permitiendo una mejora en los parámetros de calidad asistencial en su trabajo.

Como último aporte beneficioso para la población y para los profesionales que trabajan en la urgencia rural es la posibilidad de distribuir la posición o el punto geolocalizado de la urgencia por mensaje móvil ${ }^{17}$, o Whatsapp, como por ejemplo, un accidente múltiple en el que se soliciten múltiples recursos; bomberos, ambulancias o policía foral de varias zonas. Se puede derivar directamente a los recursos el archivo de coordenadas para que lo integren en su GPS o dispositivo móvil y lograr que estos acudan lo más rápidamente posible.

El Real Decreto 836/2012, de 25 de mayo, por el que se establecen las características técnicas, el equipamiento sanitario y la dotación de personal de los vehículos de transporte sanitario por carretera publicado en el Boletín Oficial del Estado Núm. 137, de 8 de junio de $2012^{18}$ dice: Las ambulancias asistenciales deberán contar, además, con dispositivos de transmisión de datos y localización GPS con su Centro de Coordinación de Urgencias. La disminución de los tiempos de atención resulta de alta importancia a la hora de valorar el resultado final sobre todo en casos graves. La disminución de los ratios de actuación permiten una mejor y más adecuada actuación de los equipos de emergencias, siendo esto también aplicable a bomberos, policías, cuerpos de seguridad, etc.

La geolocalización puede ser aplicable en todos los ámbitos de la vida tanto a nivel particular como a nivel público. Atendiendo a criterios de interés específicos, con las características específicas de cada trabajo, resuelven los problemas que se dan en la vida cotidiana de localización. Cuanta menor cobertura de telefonía o peor localización GPS se obtenga en una zona determinada, de mucha mayor utilidad resulta la geolocalización. A pesar de que a nivel par- 
ticular se utiliza en ocasiones para marcar puntos de interés, lo realmente interesante resulta de su utilización a nivel masivo para localizar avisos, pacientes, clientes, zonas... que resulten de interés estratégico para, sanitarios, empresas, organizaciones, sistemas de salud o protección civil.

Actualmente no existen referencias publicadas a la utilidad de la geolocalización en el campo sanitario asistencial. La adhesión a este sistema depende de tecnologías muy extendidas y generalizadas en la actualidad en los dispositivos móviles con tecnología GPS, además de la versatilidad que se produce al ser un sistema abierto compatible con todo tipo de plataformas. La utilización de este nuevo sistema en zonas de difícil localización y dificultosa orografía reporta importantes beneficios en el acceso al domicilio del paciente reduciendo tiempos de actuación al localizar el punto exacto del aviso urgente o emergente. Este sistema disminuye tiempos y costes, aumentando la eficiencia y el coste beneficio, produciendo un aumento importante de la calidad percibida y real.

En referencia a la cita: "preguntando se va a Roma", si se aplicase este nuevo sistema de geolocalización, esta frase dejaría de tener sentido.

\section{BIBLIOGRAFÍA}

1. Monografias.com. Comunicaciones Móviles [Internet]. Consultado en: noviembre-diciembre 2010. Disponible en: http:// www.monografias.com/especiales/comunicamov/index2.shtml

2. SERVIMEDIA. Las tics en el ámbito sanitario mejoran el acceso a la información y favorecen la inclusión social [Internet]. Mayo 2011. Disponible en: http://ecodiario.eleconomista.es/sociedad/noticias/1758894/12/09/Lastics-en-el-ambito-sanitario-mejoran-el-acceso-a-la-informacion-y-favorecen-la-inclusionsocial.html

3. Conversión de formatos ( $\mathrm{kml}$ to ov2 y viceversa) [Internet]. Febrero 2011. Disponible en: http://noticiasinteres.blogspot.com. es/2008/05/conversin-de-formatos-kml-to-ov2y.html

4. Francesc Bracero. La geolocalización se implanta en todos los ámbitos[Internet]. Agosto 2011. Disponible en: http://www.lavanguardia.com/internet/20110311/54124889951/ la-geolocalizacion-se-implanta-en-todos-losambitos.html

5. Rubén Colomer. Hipoqih, avisos georeferenciados [Internet]. Junio 2011. Disponible en: http://www.online.com.es/2119/aplicaciones-web/hipoqih-avisos-georeferenciados/

6. Rubén Colomer. Google Latitude [Internet]. Mayo 2011. Disponible en: http://www.online.com.es/3235/tecnologia/google-latitude/

7. masingenio.org. Abrir Archivos KMZ, KML, GPX en tu Android con Google Earth o Maps [Internet]. Noviembre 2010. Disponible en: http://www.masingenio.org/2011/10/abrirarchivos-kmz-kml-gpx-en-tu.html

8. Google Earth foros. Como ver un KMZ o KML en Google Maps. Diciembre 2010. Disponible en: http://www.google-earth.es/foros.php? $\mathrm{t}=4338$

9. Blog de Dr. Max Glaser. Ahora Google Maps indexa y muestra archivos en KML[Internet]. Enero 2011. Disponible en: http://www.maxglaser.net/ahora-google-maps-indexa-y-muestraarchivos-en-kml/

10. Google. Google Maps [Internet]. Disponible en: http://maps.google.es/

11. Agenciacreativa.net. Obtener coordenadas en un punto google maps [Internet]. Octubre 2010. Disponible en: http://www.agenciacreativa.net/coordenadas_google_maps.php

12. Arzur. Pasos para meter lugares en google maps. [Internet]. Enero 2011. Disponible en: http://furgonetto.wordpress.com/.

13. Google Forums. Kmz con google earth e importar con google maps [Internet]. Febrero 2011. Disponible en: http://productforums.google. com/forum/\#!topic/maps-es/DZzLXiNZi0E

14. NESUA Geo 3D. Cómo volver a descargar el KMZ de Google Maps[Internet]. Agosto 2011. Disponible en: http://geo3d.nesua. com/2011/07/como-volver-descargar-el-kmzde-google.html

15. José Miguel Ablitas. Blog de geolocalización. [Internet]. Desde mayo 2011. Disponible en: http://rekonditolugar.blogspot.com.es/

16. Google-latlong.blogspot.com. Cómo descargar mapas de Google Maps al teléfono para poder utilizarlos en modo offline, explicado al detalle [Internet]. Disponible en: http:// www.actualidadgoogle.com/2011/07/07/como-descargar-mapas-de-google-maps-al-telefono-para-poder-utilizarlos-en-modo-offlineexplicado-al-detalle/\#.T7fQcyaizfI.

17. Nishi. Android, importar y exportar mapas [Internet]. Junio 2011. Disponible en: http:// www.foromtb.com/showthread.php/610373Tutorial-Android-importar-y-exportar-mapas

18. Boletín Oficial del Estado Núm. 137, viernes 8 de junio de 2012. pp. 41589-41595. 